\title{
Proof of the generalized Lieb-Wehrl conjecture for integer indices larger than one
}

\author{
Ayumu Sugita \\ Yukawa Institute for Theoretical Physics, \\ Kitashirakawa-Oiwakecho, Kyoto, Kyoto 606-8502, Japan
}

(Dated: November 1, 2018)

\begin{abstract}
Gnutzmann and Życzkowski have proposed the Rényi-Wehrl entropy as a generalization of the Wehrl entropy, and conjectured that its minimum is obtained for coherent states. We prove this conjecture for the Rényi index $q=2,3, \ldots$ in the cases of compact semisimple Lie groups. A general formula for the minimum value is given.
\end{abstract}

PACS numbers: 02.20.Qs, 05.45.Mt 


\section{INTRODUCTION}

The Husimi function [1] is a useful tool to investigate dynamical properties of quantum systems. Wehrl proposed a classical entropy [2] using the Husimi function and conjectured that the minimum of the entropy is obtained for coherent states. His conjecture was soon proved by Lieb [3]. However, Lieb's analogous conjecture for spin coherent state in the same paper has remained unproved for more than 20 years.

The Husimi function for the density operator $\hat{\rho}$ is defined as

$$
\rho(\zeta) \equiv\langle\zeta|\hat{\rho}| \zeta\rangle
$$

where $|\zeta\rangle$ is a coherent state. The generalized coherent states defined by Perelomov [4] can also be used instead of the standard coherent state. Gnutzmann and Życzkowski [5] have recently generalized the Wehrl entropy by analogy with the well-known Rényi entropy [6]. The Rényi-Wehrl entropy is defined as

$$
S^{(q)}[\hat{\rho}]=\frac{1}{1-q} \log M^{(q)}[\hat{\rho}]
$$

Here, $M^{(q)}$ is the moment of the Husimi function

$$
M^{(q)}[\hat{\rho}]=c \int d \mu(\zeta)\{\rho(\zeta)\}^{q}
$$

where $d \mu(\zeta)$ is the Haar measure and $c$ is a normalization constant. In the limit $q \rightarrow 1$, it reproduces the usual form of the entropy

$$
\lim _{q \rightarrow 1} S^{(q)}[\hat{\rho}]=S[\hat{\rho}] \equiv-c \int d \mu(\zeta) \rho(\zeta) \log \rho(\zeta)
$$

The generalized Lieb-Wehrl conjecture formed in [5] is the following.

Conjecture 1 The minimum value of the Rényi-Wehrl entropy for $q>0$ is obtained for coherent states.

We prove this conjecture for integer indices $q \geq 2$ in the cases of the coherent states of compact semisimple Lie groups. The case of spin $(S U(2))$ coherent states has already been proved [5, 7]. In the case of the standard coherent state, $q=1$ corresponds to the original Wehrl's conjecture proved in [3], and theorem 3 therein gives the proof for arbitrary $q>1$. 


\section{GENERALIZED COHERENT STATES}

Before coming to the proof, let us review the definition and some properties of the generalized coherent states 4 . Let $G$ be a compact semisimple Lie group. The Lie algebra of $G$ can be written in so-called Cartan basis $\left\{H_{j}, E_{\alpha}\right\}$. An irreducible representation of $G$ is characterized by the lowest (or the highest) weight. Let $|-\lambda\rangle$ be the lowest weight state with the weight $-\lambda$. We denote the irreducible representation space specified by $|-\lambda\rangle$ as $D_{\lambda}$. Coherent states in $D_{\lambda}$ are obtained by the action of the group $G$ on $|-\lambda\rangle$, which can be written explicitly as $[11$

$$
|\zeta\rangle=\mathcal{N}(\zeta) \exp \left(\zeta_{\alpha} E_{\alpha}\right)|-\lambda\rangle
$$

Here, $\mathcal{N}(\zeta)$ is the normalization constant and $\alpha$ runs over all positive roots. The expansion of the exponential function becomes a finite series because $D_{\lambda}$ is finite dimensional. Therefore

$$
\tilde{\psi}(\zeta) \equiv \frac{\langle\psi \mid \zeta\rangle}{\mathcal{N}(\zeta)}
$$

is a polynomial of $\zeta$ for any state $|\psi\rangle$. We will use this fact later to prove lemma 1 .

The "resolution of unity"

$$
I_{D_{\lambda}}=\operatorname{dim} D_{\lambda} \int d \mu(\zeta)|\zeta\rangle\langle\zeta|
$$

is valid, and hence the normalization constant in (3) should be taken as

$$
c_{D_{\lambda}}=\operatorname{dim} D_{\lambda}
$$

in order to satisfy the normalization condition $M^{(1)}=1$.

\section{PROOF OF THE MAIN RESULT}

Turning now to the proof, we first note the following lemma.

Lemma 1 If $\hat{\rho}$ minimizes $S^{(q)}[\hat{\rho}]$ for some $q>0$, $\hat{\rho}$ must be a pure state.

This is a generalization of lemma 2 in [3], and can be proved in the same way (see the appendix). Hereafter we concentrate on pure states. 
Let us consider the simplest case $q=2$. We will see later that the discussion in this case can be easily generalized. We have to prove that the maximum of the second moment of the Husimi function

$$
M_{|\varphi\rangle}^{(2)} \equiv c_{D_{\lambda}} \int d \mu(\zeta)|\langle\zeta \mid \varphi\rangle|^{4}
$$

is given by coherent states.

Let us rewrite (9) as

$$
M_{|\varphi\rangle}^{(2)}=\left(\left\langle\varphi|\otimes\langle\varphi|) c_{D_{\lambda}} \int d \mu(\zeta)(|\zeta\rangle \otimes|\zeta\rangle)(\langle\zeta|\otimes\langle\zeta|)(|\varphi\rangle \otimes|\varphi\rangle) .\right.\right.
$$

The key observation is that the tensor product of the coherent states $|\zeta\rangle \otimes|\zeta\rangle$ is a coherent state in the irreducible representation space $D_{2 \lambda}$ whose lowest weight state is $|-\lambda\rangle \otimes|-\lambda\rangle$. The same idea is used in [0] for $S U(2)$. It can be shown explicitly as

$$
\begin{aligned}
& \left(H_{j} \otimes I+I \otimes H_{j}\right)|-\lambda\rangle \otimes|-\lambda\rangle=-2 \lambda_{j}|-\lambda\rangle \otimes|-\lambda\rangle \\
& \left(E_{-\alpha} \otimes I+I \otimes E_{-\alpha}\right)|-\lambda\rangle \otimes|-\lambda\rangle=0 \\
& |\zeta\rangle \otimes|\zeta\rangle=\mathcal{N}(\zeta)^{2} \exp \left[\zeta_{\alpha}\left(E_{\alpha} \otimes I+I \otimes E_{\alpha}\right)\right]|-\lambda\rangle \otimes|-\lambda\rangle
\end{aligned}
$$

where $\alpha$ runs over positive roots. Therefore $\int d \mu(\zeta)(|\zeta\rangle \otimes|\zeta\rangle)(\langle\zeta| \otimes\langle\zeta|)$ is proportional to the projection operator to $D_{2 \lambda}$ in $D_{\lambda} \otimes D_{\lambda}$, i.e.

$$
c_{D_{\lambda}} \int d \mu(\zeta)(|\zeta\rangle \otimes|\zeta\rangle)(\langle\zeta| \otimes\langle\zeta|)=\frac{\operatorname{dim} D_{\lambda}}{\operatorname{dim} D_{2 \lambda}} P_{D_{2 \lambda}}
$$

Here the proportional constant is determined from (7). Hence

$$
M_{|\varphi\rangle}^{(2)}=\frac{\operatorname{dim} D_{\lambda}}{\operatorname{dim} D_{2 \lambda}}\left(\left\langle\varphi|\otimes\langle\varphi|) P_{D_{2 \lambda}}(|\varphi\rangle \otimes|\varphi\rangle)\right.\right.
$$

and we have

$$
|\varphi\rangle \otimes|\varphi\rangle \in D_{2 \lambda} \Longleftrightarrow M_{|\varphi\rangle}^{(2)}=M_{\max }^{(2)} \equiv \frac{\operatorname{dim} D_{\lambda}}{\operatorname{dim} D_{2 \lambda}}
$$

Then we should prove

$$
|\varphi\rangle \text { : coherent state } \Longleftrightarrow|\varphi\rangle \otimes|\varphi\rangle \in D_{2 \lambda} \text {. }
$$

LHS $\Rightarrow$ RHS is obvious, because $|\varphi\rangle \otimes|\varphi\rangle$ is obtained by the action of $G$ on $|-\lambda\rangle \otimes|-\lambda\rangle$ which belongs to $D_{2 \lambda}$ by definition. 
Then we prove LHS $\Leftarrow$ RHS. Let us consider the quadratic Casimir operator $C_{2}$ of the group $G$. By taking an orthonormal basis $\left\{T_{a}\right\}$ of the Lie algebra, it can be written as

$$
C_{2}=\boldsymbol{T}^{2} \equiv \sum_{a} T_{a}^{2}
$$

In the tensor product space $D_{\lambda} \otimes D_{\lambda}$, the quadratic Casimir operator is written as

$$
C_{2}^{(2)} \equiv \sum_{a}\left(T_{a} \otimes 1+1 \otimes T_{a}\right)^{2}=\boldsymbol{T}^{2} \otimes 1+1 \otimes \boldsymbol{T}^{2}+2 \sum_{a} T_{a} \otimes T_{a}
$$

Therefore

$$
\left(\left\langle\varphi|\otimes\langle\varphi|) C_{2}^{(2)}(|\varphi\rangle \otimes|\varphi\rangle)=2<\boldsymbol{T}^{2}>+2<\boldsymbol{T}\right\rangle^{2}\right.
$$

where the symbol $<>$ denotes the expectation value for $|\varphi\rangle$.

Here we note the lemma proved in [8]:

Lemma 2 (Delbourgo and Fox) The minimum of the uncertainty $(\Delta T)^{2} \equiv<\boldsymbol{T}^{2}>-<$ $\boldsymbol{T}>^{2}$ is obtained for coherent states

$$
(\Delta T)^{2} \text { is minimum } \Longleftrightarrow|\varphi\rangle \text { : coherent state. }
$$

Since $C_{2}=\boldsymbol{T}^{2}$ is a constant in $D_{\lambda},\langle\boldsymbol{T}\rangle^{2}$ is maximum when $|\varphi\rangle$ is a coherent state, and hence $C_{2}^{(2)}$ takes the maximum value at $D_{2 \lambda}$ because it contains the tensor product of the coherent states. Therefore

$$
|\varphi\rangle \otimes|\varphi\rangle \in D_{2 \lambda} \Longrightarrow\left\langle\boldsymbol{T}>^{2} \text { is maximum } \Longrightarrow \mid \varphi\right\rangle: \text { coherent state. }
$$

This completes the proof of (17).

The generalization to the higher moments is straightforward. By considering the tensor product space $D_{\lambda}^{\otimes q} \equiv \overbrace{D_{\lambda} \otimes D_{\lambda} \otimes \ldots \otimes D_{\lambda}}^{q}$, one can easily show

$$
M_{|\varphi\rangle}^{(q)} \equiv \frac{\operatorname{dim} D_{\lambda}}{\operatorname{dim} D_{q \lambda}}\left(\left\langle\left.\varphi\right|^{\otimes q}\right) P_{D_{q \lambda}}\left(|\varphi\rangle^{\otimes q}\right)\right.
$$

and

$$
\left(\left\langle\left.\varphi\right|^{\otimes q}\right) C_{2}^{(q)}\left(|\varphi\rangle^{\otimes q}\right)=q<\boldsymbol{T}^{2}>+q(q-1)<\boldsymbol{T}>^{2}\right.
$$

in obvious notation. From these two formulae we can conclude

$$
M_{|\varphi\rangle}^{(q)}=M_{\max }^{(q)} \Longleftrightarrow|\varphi\rangle^{\otimes q} \in D_{q \lambda} \Longleftrightarrow|\varphi\rangle: \text { coherent state. }
$$

Combining this and Lemma 1, we obtain the main result of this paper: 
Theorem 1 Let $S^{(q)}$ be the Rényi-Wehrl entropy with an integer index $q \geq 2$ of a compact semisimple Lie group. The minimum value of $S^{(q)}$ is obtained for coherent states

$$
S^{(q)}[\hat{\rho}] \text { is minimum } \Longleftrightarrow \hat{\rho}=|\varphi\rangle\langle\varphi|,| \varphi\rangle \text { : coherent state. }
$$

The minimum value $S_{\text {min }}^{(q)}$ is given by

$$
S_{\text {min }}^{(q)}=\frac{1}{1-q} \log \left(\frac{\operatorname{dim} D_{\lambda}}{\operatorname{dim} D_{q \lambda}}\right)
$$

where $D_{\lambda}$ is the irreducible representation space in which $S^{(q)}$ is defined.

\section{MINIMUM VALUES FOR SOME SPECIAL CASES}

Finally, let us examine the general formula for the minima (27) in some special cases. Cases (i), (ii), and (iii) in the following can be used to investigate the dynamics of bosons, fermions and distinguishable particles, respectively [12] [9].

(i) $G=S U(N), D_{\lambda}=\left[1^{m}\right]$ (symmetric product of $m$ fundamental representations)

$$
\begin{aligned}
& \operatorname{dim} D_{q \lambda}=\operatorname{dim}\left[1^{q m}\right]=\frac{(N+q m-1) !}{(N-1) !(q m) !} \\
& S_{\text {min }}^{(q)}=\frac{1}{1-q} \log \frac{\Gamma(N+m) \Gamma(q m+1)}{\Gamma(N+q m) \Gamma(m+1)} .
\end{aligned}
$$

Assuming that (29) holds also for non-integer $q$, we obtain

$$
\lim _{q \rightarrow 1} S_{\min }^{(q)}=m\{\psi(N+m)-\psi(m+1)\}
$$

where $\psi(z) \equiv \frac{d}{d z} \log \Gamma(z)$ is the digamma function. This result agrees with the value of $S^{(1)}$ for coherent states given in [10], except for the shift by $-\ln N$ due to another normalization of the coherent states.

(ii) $G=S U(N), D_{\lambda}=[m]$ (anti-symmetric product of $m$ fundamental representations)

$$
\begin{aligned}
& \operatorname{dim} D_{q \lambda}=\operatorname{dim}\left[m^{q}\right]=\prod_{j=0}^{m-1} \frac{(N+q-j-1) ! j !}{(N-j-1) !(q+j) !} \\
& S_{\text {min }}^{(q)}=\frac{1}{1-q} \sum_{j=0}^{m-1} \log \frac{\Gamma(N+1-j) \Gamma(q+j+1)}{\Gamma(N+q-j) \Gamma(j+2)} .
\end{aligned}
$$

In this case, the minimum value for $q=1$ is expected to be

$$
\lim _{q \rightarrow 1} S_{\min }^{(q)}=\sum_{j=0}^{m-1}\{\psi(N-j+1)-\psi(j+2)\} .
$$


(iii) $G=\overbrace{S U(N) \times \ldots \times S U(N)}^{m}, D_{\lambda}=[1]^{\otimes m}$

$$
\begin{aligned}
\operatorname{dim} D_{q \lambda} & =\operatorname{dim}\left[1^{q}\right]^{\otimes m}=\left(\frac{(N+q-1) !}{(N-1) ! q !}\right)^{m} \\
S_{\text {min }}^{(q)} & =\frac{m}{1-q} \log \frac{\Gamma(N+1) \Gamma(q+1)}{\Gamma(N+q)} .
\end{aligned}
$$

The minimum value for $q=1$ is expected to be

$$
\lim _{q \rightarrow 1} S_{\min }^{(q)}=m\{\psi(N+1)-\psi(2)\} .
$$

\section{Acknowledgments}

The author thanks Professor Gnutzmann for letting him know about reference [7]. He also thanks Professor Życzkowski for helpful discussions.

\section{APPENDIX A: PROOF OF LEMMA 1}

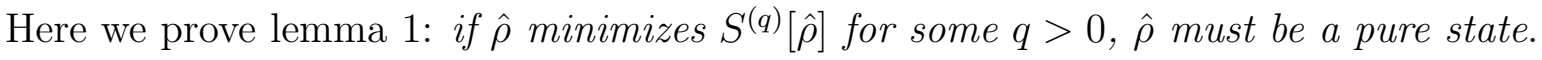

We consider three cases (i) $0<q<1$, (ii) $q=1$ and (iii) $1<q$ separately. $\hat{\rho}$ minimizes $M^{(q)}$ and $S$ in cases (i) and (ii), respectively, and maximizes $M^{(q)}$ in case (iii). Let us decompose the density operator as $\hat{\rho}=\sum_{i} \lambda_{i} \hat{\rho}_{i}$, where $\sum_{i} \lambda_{i}=1$ and $\hat{\rho}_{i} \equiv\left|\psi_{i}\right\rangle\left\langle\psi_{i}\right|$ is a pure state. Since $m^{(q)}(x)=x^{q}(0<q<1)$ and $s(x)=-x \log x$ are concave, and $m^{(q)}(x)=x^{-q}(q>1)$ is convex,

$$
\begin{aligned}
M^{(q)}[\hat{\rho}] & \geq \sum_{i} \lambda_{i} M^{(q)}\left[\hat{\rho}_{i}\right] \quad(0<q<1) \\
S[\hat{\rho}] & \geq \sum_{i} \lambda_{i} S\left[\hat{\rho}_{i}\right] \\
M^{(q)}[\hat{\rho}] & \leq \sum_{i} \lambda_{i} M^{(q)}\left[\hat{\rho}_{i}\right] \quad(q>1) .
\end{aligned}
$$

The equality must hold in at least one of the three inequalities. Then $\rho_{i}(\zeta)=\rho_{j}(\zeta)$, and hence $\left|\tilde{\psi}_{i}(\zeta)\right|=\left|\tilde{\psi}_{j}(\zeta)\right|$ in the notation of (6), almost everywhere for all $i, j$. Since $\tilde{\psi}_{i}$ is a polynomial, $\tilde{\psi}_{i}=a \tilde{\psi}_{j}$, and hence $\left|\psi_{i}\right\rangle=a^{*}\left|\psi_{j}\right\rangle$, with $|a|=1$. Therefore $\hat{\rho}_{i}=\hat{\rho}_{j}$ for all $i, j$, which means $\hat{\rho}$ is a pure state.

[1] K. Husimi, Proc. Phys. Math. Soc. Japan 22264 (1940). 
[2] A. Wehrl, Reps. Math. Phys. 16, 353 (1979).

[3] E. H. Lieb, Commun. Math. Phys. 62, 35 (1978).

[4] A. Perelomov, Generalized Coherent States and Their Applications, Springer-Verlag Berlin Heidelberg (1986).

[5] S. Gnutzmann and K. Życzkowski, J. Phys. A 34 (2001) 10123.

[6] A. Rényi, Probability Theory, North-Holland, Amsterdam (1970).

[7] P. Schupp, Commun. Math. Phys. 207 (1999) 481.

[8] R. Delbourgo and J. R. Fox, J. Phys. A 10 (1977) L233.

[9] A. Sugita, arXiv: nlin.CD/0112042

[10] W. Słomczyński and K. Życzkowski, Phys. Rev. Lett. 80 (1998) 1880.

[11] In fact, (5) cannot represent the coherent states orthogonal to $|-\lambda\rangle$. However, it does not matter when we consider entropies such as (2) because the measure of the set of such states is zero.

[12] $U(N)$, instead of $S U(N)$, is used in 9 for bosons and fermions. $U(N)$ is not semisimple. However, $U(N) \sim U(1) \times S U(N)$, and the Abelian subgroup $U(1)$ corresponds to the total phase which is irrelevant for the Husimi functions. 